\title{
Demand forecasting for companies with many branches, low sales numbers per product, and non-recurring orderings
}

\author{
Sascha Kurz and Jörg Rambau \\ University of Bayreuth \\ Departement of Mathematics \\ 95440 Bayreuth, Germany \\ \{sascha.kurz,jörg.rambau\}@uni-bayreuth.de
}

\begin{abstract}
We propose the new Top-Dog-Index to quantify the historic deviation of the supply data of many small branches for a commodity group from sales data. On the one hand, the common parametric assumptions on the customer demand distribution in the literature could not at all be supported in our real-world data set. On the other hand, a reasonably-looking non-parametric approach to estimate the demand distribution for the different branches directly from the sales distribution could only provide us with statistically weak and unreliable estimates for the future demand. Based on real-world sales data from our industry partner we provide evidence that our Top-Dog-Index is statistically robust. Using the Top-Dog-Index, we propose a heuristics to improve the branch-dependent proportion between supply and demand. Our approach cannot estimate the branchdependent demand directly. It can, however, classify the branches into a given number of clusters according to an historic oversupply or undersupply. This classification of branches can iteratively be used to adapt the branch distribution of supply and demand in the future.
\end{abstract}

\section{Introduction}

Many retailers have to deal in their daily businesses with small profit margins. Their economic success lies mostly in the ability to forecast the customers' demand for individual products. More specifically: trade exactly what you can sell to your customers. This task has two aspects if your company has many branches in different regions: trade what your customers would like to buy because the product as such is attractive to them and provide a demand adjusted number of items for each branch or region.

In this paper we deal with the second aspect only: meet the branch distributed demand for products as closely as possible. The first aspect clearly also interferes with the total demand for a product over all branches. Therfore, we assume that we are given a fix total number of items per product which should be distributed over the set of branches to meet the the branch-dependent demand distribution as closely as possible.

Our industry partner is a fashion discounter with more than 1000 branches most of whose products are never replenished, except for the very few "never-out-of-stock"products (NOS products): because of lead times of around three months, apparel replenishments would be too late anyway. In most cases the supplied items per product and apparel size lie in the range between 1 and 6 .

The task can be formulated informally as follows: Given historic supply and sales data for a commodity group, find out some robust information on the demand distribution over branches in that commodity group that can be used to optimize or at least to improve the supply distribution over all branches.

We remark that trading fashion has the special feature that also the demand for different apparel size varies over the branches. In this article, however, we focus on the aspect of improving the supply distribution over all branches. The apparel size distribution problem is subject some other research in progress.

\section{$1.1 \quad$ Related work}

Demand forecasting for NOS items is an well-studied topic both in research and practice. The literature is overboarding, see, e.g., $[1,2,3]$ for some surveys. For promotional items and other items with single, very short life cycles, however, we did not find any suitable demand forecasting methods.

The literature in revenue management (assortment optimization, inventory control, dynamic pricing) very often assumes the neglectability of out-of-stock substitution effects. This out-of-stock substitution in the sales data of our 
partner, however, poses the biggest problem in our case. In our real-world application we have no replenishment, small volume deliveries per branch, lost sales with unknown or even no substitution, sales rates depending much more on the success of the individual product at the time it was offered than on the size. Therefore, estimating the absolute future demand distribution from historical sales data with no correction for out-of-stock substitution seems questionable.

Most demand forecasting tools used in practice are provided by specialized software companies. Quite a lot of software packages are available, see [6] for an overview. Our partner firm has checked several offers in the past and did - apart from the NOS segment — not find any optimization tools tailored to their needs.

\subsection{Our contribution}

We show that a reasonably-looking attempt to measure the demand distribution over all branches by measuring for each branch the sales over all products up to a certain day (to avoid out-of-stock substitution) does not work because of the high volatility in the sales rates of different products.

The key idea of this work is that estimating something weaker than the absolute fraction of total demand of a branch will result in stronger information that is still sufficient to improve on the demand consistency of the supply of branches.

More specifically, we propose the new Top-Dog-Index (TDI) that can measure the branch dependent deviation of demand from supply, even for very small sales amounts or short selling periods. This yields, in particular, an estimate for the direction in which the supply was different from demand in the past for each branch.

On the one hand, the TDI is a rather coarse measurement; on the other hand, we can show that on our real-world data set it is statistically robust in the sense that the TDIs of the branches relative to each other are surprisingly similar on several independent samples from the sales data and their complements.

To show the value of the information provided by the TDI, we propose a dynamic optimization procedure that shifts relative supply among branches until the deviation measured is as small as possible.

Of course, the impact of such an optimizaton procedure has to be evaluated in practice. This is subject of future research.

\subsection{Outline of the paper}

In Section 2 we state the real-world problem we are interested in. Moreover, we give an abstract problem formulation. An obvious approach of determining the demand distribution of the branches directly from historic sales data is shown to be inappropriate on our given set of sales data in Section 3. We propose our new Top-Dog-Index in Section 4 . We analyze its statistical robustness and its distinctive character in clustering branches according to the deviation of the historic ratio between supply and demand. In Section 5 we describe an heuristic iterative procedure that uses the information from the Top-Dog-Indices to alter the supply distribution towards a suitable distribution that more or less matches the demand distribution over branches. An outlook and a conclusion will be given in Section 6 .

\section{The real-world problem and an abstract problem formulation}

Our industry partner is a fashion discounter with over 1000 branches. Products can not be replenished and the number of sold items per product and branch is rather small. There are no historic sales data for a specific product available since every product is sold only for one selling period. The challenge for our industry partner is to determine a suitable total amount of items of a specific product which should be bought. For this part the knowledge and experience of the buyers employed by a fashion discounter is used. We seriously doubt that a software package based on historic sales data can do better. But there is another task being more accessible for computer aided forecasting methods. Once the total amount of sellable items of a specific product is determined, one has to decide how to distribute this total amount to a set of branches $B$ which differ in their demand. The remaining part of this paper addresses the latter task.

In the following, we formulate this problem in a more abstract way. Given a set of branches $B$, a set of products $P$, a function $S(b, p)$ which denotes the historic supply of product $p$ for each branch $b$, and historic sales transactions from which one can determine how many items of a given product $p$ are sold in a given branch $b$ at a given day of sales $d$. The target is to estimate a demand $\eta(b, \tilde{p})$ for a future product $\tilde{p} \notin P$ in a given branch $b$, where we can use $\sum_{b \in B} \eta(b, \tilde{p})=1$ as normalization. This estimation $\eta(b, \tilde{p})$ should be useable as a good advice for a supply $S(b, \tilde{p})$. No further information, e.g., on a stochastic model for the purchaser behavior, is available.

\section{Some real-data analysis evaluating an obvi- ous approach}

The most obvious approach to determine a demand distribution over branches is to count the sold items per branch and divide by the total number of sold items. Here we have some freedom to choose the day of the sale where we mea- 
sure these magnitudes. We have to balance two competing influences. An early measurement may provide numbers of sale which are statistically too small for a good estimate. On the other hand on a late day of sales there might be too much unsatisfied demand to estimate the demand since no replenishment is possible in our application.

The business strategy of our partner implies to cut prices until all items are sold. So, a very late measurement would only estimate the supply instead of the demand. As there is no expert knowledge to decide which is the optimal day of sales to measure the sales and estimate the branch dependent demand distribution we have adapted a statistical test to measure the significance of the demand distributions obtained for each possible day of counting the sold items. Given a data set $D$, a day of sales $d$ let $\phi_{b, d}(D)$ be the estimated demand for branch $b$ determined using the amounts of sold items up to day $d$ as described above.

We normalize the values $\phi_{b, d}(D)$ so that we have $\sum_{b \in B} \phi_{b, d}(D)=1$ for each day of sales $d$, where $B$ is the set of branches. A common statistical method to analyze the reliability of a prediction based on some data universe $D$ is to randomly partition $D$ into two nearly equally sized disjoint samples $D_{1}$ and $D_{2}$ with $D_{1} \cup D_{2}=D$ and to compare the prediction based on $D_{1}$ with the prediction based on $D_{2}$. If the two predictions differ substantially than the used prediction method is obviously not very trustworthy or statistically speaking not very robust.

In the following part of this section we analyze the robustness of the prediction $\phi_{b, d}(D)$ for every possible sales day, meaning that even an optimal sales day for the measurement does not provide a prediction being good enough for our purpose. To measure exactly by how much two predictions $\phi_{\cdot, d}\left(D_{1}\right)$ and $\phi_{\cdot, d}\left(D_{2}\right)$ differ we introduce the following:

Definition 1 For a given sales day $d$ and two samples $D_{1}$ and $D_{2}$ we define the discrepancy $\delta_{d}$ as

$$
\delta_{d}\left(D_{1}, D_{2}\right):=\sum_{b \in B}\left|\phi_{b, d}\left(D_{1}\right)-\phi_{b, d}\left(D_{2}\right)\right| .
$$

Similarly we define a discrepancy between supply and demand. We compare both discrepancies in Figure 1. The result: there is no measuring day for which the discrepancy between two samples is smaller than the discrepancy between a sample and the supply. In other words, if we consider the discrepancy between supply and demand as a measure for the inconsistency of the supply distribution with the demand distribution, then either the supply is not significantly inconsistent with demand (i.e., we should better change nothing) or the measurements on the various samples are significantly different (i.e., nothing can be learned about how to correct the supply distribution).

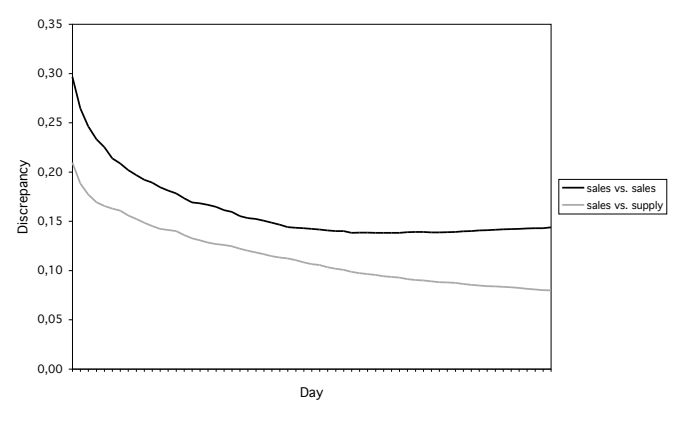

Figure 1. Discrepancy for the first 60 days.

An explanation why this obvious approach does not work well in our case is due to the small sale numbers and the interference of the demand of a branch with product attractivity and price cutting strategies. In Figure 2 we depict the change of prediction $\phi_{\mathbf{b}, \mathbf{d}}(\mathbf{D})$ over time for five characteristic but arbitrary branches

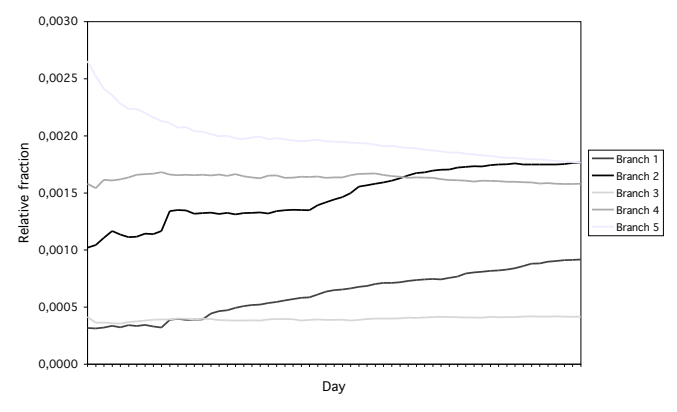

Figure 2. Prediction $\phi_{\mathbf{b}, \mathbf{d}}(\mathbf{D})$ over time.

We would like to remark that one of the authors currently advises two diploma theses which check some common parametric models for demand forecasting on historic sales data from literature. None of them gives significant information of the demand distribution over branches of our data set because the data does not exhibit any similarity to the parametric distributions coming from economic theory and the like. This may be due to the fact that the contaminating effects of promotion, mark-downs, openings/closings of competing stores prohibit a causal model for the demand. We do not claim that the assumptions of parametric demand models never hold, but in our application they are most certainly not met.

\section{The Top-Dog-Index (TDI)}

In the previous section we learned that in our application we cannot utilize the most obvious approach of looking at the sales distribution over the different branches on 
an arbitrary but fixed day of the selling period of each individual product. Since there is also no indication that any of the common parametric models for the demand estimation directly from sales data fit in our application we make no assumptions on a specific stochastic distribution of the purchaser behavior.

Our new idea dismisses the desire to estimate an absolute percental demand distribution for the branches. Instead we develop an index measuring the relative success of a branch in the competition of all branches that can be estimated from historic sales data in a stable way.

To motivate our distribution free measurement we consider the following thought experiment. For a given branch $b$ and given product $p$ let $\theta_{b}(p)$ denote the stock-out-day. Let us assume that we have $\theta_{b}(p)=\theta_{b^{\prime}}(p)$ for all products $p$ and all pairs of branches $b, b^{\prime}$. In this situation one could certainly say that the branch-dependent demand is perfectly matched by the supply. Our measure tries to quantify the variation of the described ideal situation.

Therefore, we sort for each product $p$ the stock-out-days $\theta_{b}(p)$ in increasing order. If for a fixed product $p$ a branch $b$ is among the best third according to this list it gets a winning point for $p$. If it is among the last third it is assigned a losing point for $p$. With $B_{p}$ being the set of branches which trade product $p$ and $P$ being the set of the products traded by the company we can define more precisely:

Definition 2 Let $b$ be a branch. The Top-Dog-Count is defined as $W(b):=$

$$
\left|\left\{p \in P\left|\frac{1}{3}\right| B_{p}|\geq|\left\{b^{\prime} \in B_{p} \mid \theta_{b^{\prime}}(p) \leq \theta_{b}(p)\right\} \mid\right\}\right|
$$

and the Flop-Dog-Count is defined as $L(b):=$

$$
\left|\left\{p \in P\left|\frac{1}{3}\right| B_{p}|\geq|\left\{b^{\prime} \in B_{p} \mid \theta_{b^{\prime}}(p) \geq \theta_{b}(p)\right\} \mid\right\}\right| .
$$

For a fix dampening parameter $C>0$ let

$$
T D I(b):=\frac{W(b)+C}{L(b)+C}
$$

be the Top-Dog-Index (TDI) of branch $b$.

If the TDI of a branch $b$ is significantly large compared to the TDIs of the other branches then we claim that branch $b$ was undersupplied in the past. Similarly, if the TDI of branch $b$ is significantly small compared to the TDIs of the other branches then we claim that branch $b$ was oversupplied in the past. We give an heuristic optimization procedure past on this information in the section. The effect of the dampening parameter $C$ is on the one hand that the TDI is well defined since division by zero is circumvented. On the other hand, and more important, the influence of small Top-Dog- or Flop-Dog-Counts, which are statistically unstable, is leveled to a decreased importance.

\subsection{Statistical significance of the TDI}

Similarly as in Section 3 we want to analyze the significance of the proposed Top-Dog-Index on some real sales data. Instead of two data sets $D_{1}$ and $D_{2}$ we use seven such samples $D_{i}$. Therefore we assign to each different product $p \in P$ a equi-distributed random number $r_{p} \in\{1,2,3,4\}$. The samples $D_{i}$ are composed as summarized in Table 1 .

\begin{tabular}{l|l}
\hline$D_{1}:=\{p \mid \in P$ & $\left.r_{p} \in\{1,2\}\right\}$ \\
$D_{2}:=\{p \mid \in P$ & $r_{p} \in\{3,4\}$ \\
$D_{3}:=\{p \mid \in P$ & $r_{p} \in\{1,3\}$ \\
$D_{4}:=\{p \mid \in P$ & $r_{p} \in\{2,4\}$ \\
$D_{5}:=\{p \mid \in P$ & $\left.r_{p} \in\{3\}\right\}$ \\
$D_{6}:=\{p \mid \in P$ & $\left.r_{p} \in\{1,2,4\}\right\}$ \\
$D_{7}:=\{p \mid \in P$ & $\left.r_{p} \in\{1,2,3,4\}\right\}$ \\
\hline
\end{tabular}

Table 1. Assignment of test sets.

For the interpretation we remark that the pairs $\left(D_{1}, D_{2}\right)$, $\left(D_{3}, D_{4}\right)$, and $\left(D_{5}, D_{6}\right)$ are complementary. The whole data population is denoted by $D_{7}$ and equals $P$. We use $T D I\left(b, D_{i}\right)$ as an abbreviation of $T D I(b)$ where $P$ is replaced by $D_{i}$.

Since the Top-Dog-Index is designed as a nonquantitative index we have to use another statistical test to assure ourselves that it gives some significant information. We find it convincing to regard the Top-Dog-Index as significant and robust whenever we have

$$
\frac{T D I\left(b, D_{i}\right)}{T D I\left(b, D_{j}\right)} \approx \frac{T D I\left(b^{\prime}, D_{i}\right)}{T D I\left(b^{\prime}, D_{j}\right)}
$$

for each pair of branches $b, b^{\prime}$ and each pair of samples $D_{i}, D_{j}$. In words we claim that the Top-Dog-Index is a relative index which is independent of the underlying sample if we consider a fixed universe $D_{7}$.

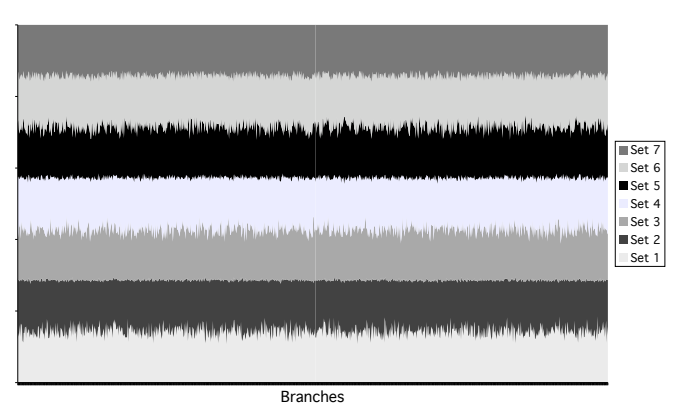
Figure 3. Relative distribution of the Top- Dog-Index on different data samples and branches.


Our first aim is to provide evidence that the $T D I(b)$ values are robust measurements. There is a nice way to look at equation (5) graphically. For each branch $b$ let us plot a column of the the relative values $\frac{T D I\left(b, D_{i}\right)}{\sum_{j} T D I\left(b, D_{j}\right)}$ for all $i$. The result for our data set is plotted in Figure 3.

To get the correct picture in the interpretation of the plot of Figure 3 we compare it to the extreme cases of deterministic numbers (i.e., $\frac{T D I\left(b, D_{i}\right)}{T D I\left(b, D_{j}\right)}=c_{i j}=c$ for all $i$ and $j$ ), see Figure 4, and random numbers, see Figure 5.

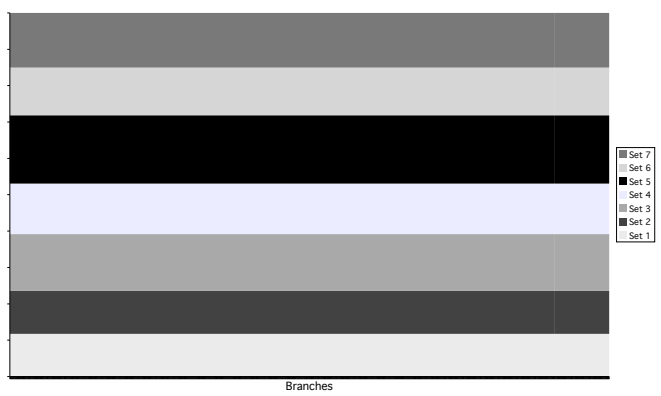

\section{Figure 4. Relative distribution of determinis- tic numbers.}

As a matter of fact, the regions of same color in the plot of the relative distribution of deterministic numbers in Figure 4 are formed by perfect rectangles, which are not forced in general to have equal height.

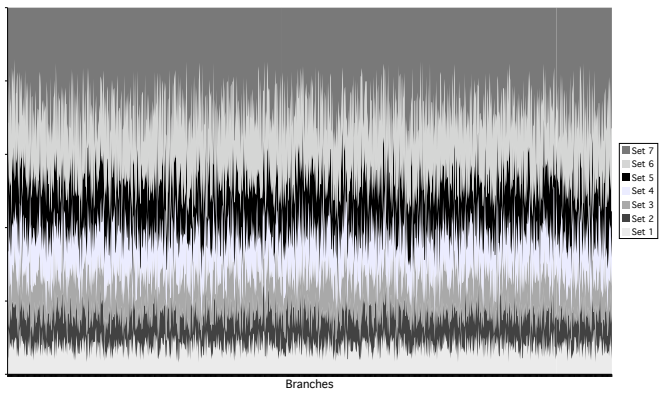

Figure 5. Relative distribution of in $[0.5,1.5]$ equi-distributed random variables.

As an example for a random plot we depict in Figure 5 the relative distribution of random numbers being equidistributed in the interval $[0.5,1.5]$.

In the plots of Figure 3, 4, and 5 we can see that that the TDI on the given data set behaves more like a perfect deterministic estimation than a random number distribution. (Ideally, one should now quantify how large the probability is to obtain a TDI chart as in Figure 3 by a random measurement.) So there is empirical evidence that the TDI gives some stable information. As a comparison of the TDI and the method described in Section 3 we depict the corresponding relativ distribution for measuring day 5 in Figure 6. Although a measurement on this day was the best we could find, it still produces more severe outliers than the TDI measurement.

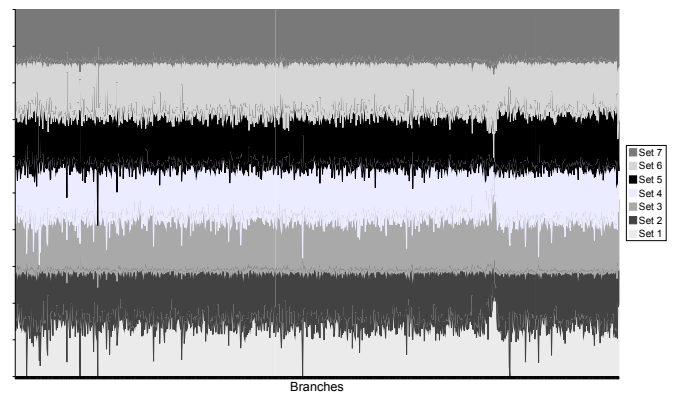

Figure 6. Relative distribution of $\phi_{\cdot, 5}$.

Now the question remains whether this information is enough to cluster branches into oversupplied and undersupplied ones. More directly: is the distinctive character of the TDI strong enough? We consider this question in the next subsection. How the TDI information can be used to iteratively improve the branch dependent ratio between supply and demand will be the topic of Section 5 .

\subsection{The distinctive character of the TDI}

If one forces the values of the TDIs to be contained in an interval of small length, then clearly a plot of the relative distributions would look like the plot of Figure 4. As an thought experiment just imagine how Figure 5 would look like, if we would use random numbers being equidistributed in the interval $[0.9,1.1]$ instead of being equidistributed in the interval $[0.5,1.5]$

Forcing the possible values of the TDIs in an interval of small length is feasible by choosing a sufficiently large dampening parameter $C$. So this parameter has to be chosen with care. We remind ourselves that we would like to use the TDIs to cluster branches. Therefore the TDIs should vary over a not to small range of values to have a good distinctive character. Clearly by using the TDI we can only detect possible improvements if the supply versus demand ratio actually inadequate in a certain level. In Figure 7 we have plotted the occurring TDIs of our data set to demonstrate there is indeed some variation of values in our data set, no matter which sample we consider (let alone the data universe). As one can see the TDIs vary widely enough to distinguish between historically under- and oversupplied branches. 


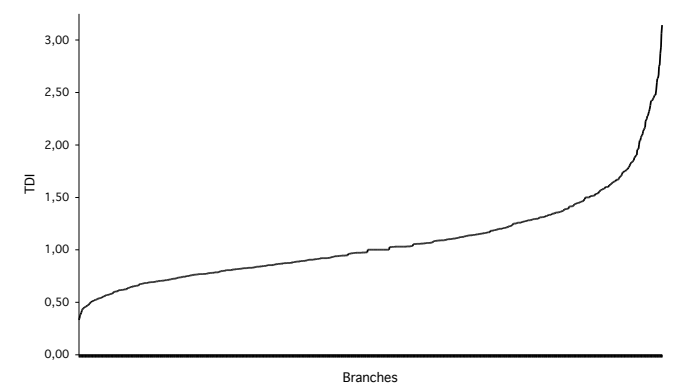

Figure 7. Occurring TDls.

\section{The heuristic supply optimization proce- dure based on the TDI}

So far we have developed and statistically stable index capturing the deviation of supply from demand for each branch. Now we have to specify how we can use this information to improve the branch dependent ratio between supply and demand.

Let $S(b)$ be the historic supply of branch $b$ being normalized so that we have $\sum_{b \in B} S(b)=1$. Our aim is to estimate supplies $\tilde{S}(b)$, also fullfilling $\sum_{b \in B} \tilde{S}(b)=1$, which are more appropriate concerning the satisfaction of demand by using the TDI information.

Therefore let us partition the interval $(0, \infty)$ of the positive real numbers into a given number of $l$ appropriate chosen intervals $\mathcal{I}_{j}$. Further we need $l$ appropriately chosen increment numbers $\Delta_{j}$. Our proposed update formula for the estimated branch dependent demand is given by

$$
\tilde{S}(b)=\frac{S(b)+\Delta_{j(b)}}{\sum_{b^{\prime} \in B} S\left(b^{\prime}\right)+\Delta_{j\left(b^{\prime}\right)}}
$$

for all branches $b$, where $j(b)$ is the unique index with $T D I(b) \in \mathcal{I}_{j(b)}$.

We do not claim that the $\tilde{S}(b)$ are a good estimation for the demand of all branches. Our claim is that they approach a good estimation of the branch dependent demand if one iterates the described procedure over several rounds and carefully chooses the increment numbers $\Delta_{j}$, which may vary over the time.

Once you have a new proposal $\tilde{S}(b)$ of the relative supply for each branch $b$, one only has to fit it into an integer valued supply for each new product $p^{\prime}$. Given the problem of apparel size assortment and pre-packing, this is easier said than done and is subject of further studies.

In contrast to the other sections here we are somewhat imprecise and there is a lot of freedom, e.g., how to choose the intervals $\mathcal{I}_{j}$ and increment numbers $\Delta_{j}$. That is for several reasons. On the one hand that is exactly the point where some expert form the business should calibrate the parameters to specific data of the company. One the other hand there are quite a lot of possibilities how to do it in detail. Their analysis will be a topic of future research. For the practical application we account rather simple than sophisticated variants in the first step.

\section{Conclusion and outlook}

We have introduced the new Top-Dog-Index which is capable to cluster branches of a retail company into oversupplied and undersupplied branches at a statistically robust niveau level where more direct methods fail. The robustnest of this method is documented by some statistical tests based on real-world data.

We have also documented that the distinctive character of the proposed TDI is significant for our application: for the first time we can gain information about the demand distribution of branches from historic sales data on only few products with volatile success in sales rates and with unknown stock-out substitution effects, and this information does not depend too much on the sample of the sales data universe out of which the TDI is computed.

For the dynamic optimization of the supply distribution among branches, some fine tuning of parameters is needed; for a real-world implementation these details have to be fixed. This, together with a field study of the impacts of an improved supply distribution are research in progress.

\section{References}

[1] J. Armstrong, editor. Principles of Forecasting: A Handbook for Researchers and Practitioners. Kluwer, 2001.

[2] A. Kok and M. Fisher. Demand estimation and assortment optimization under substitution: Methodology and application. Operations Research, to appear.

[3] S. Makridakis, S. Wheelwright, and R. Hyndman. Forecasting:Methods and Applications. Wiley, 2004.

[4] M. Mantrala and S. Rao. A decision-support system that helps retailers decide order quantities and markdowns for fashion goods. Interfaces, 31(3b):146-165, 2001.

[5] B. Pashigian. Demand uncertainty a sales: A study of fashion and markdown pricing. The American Economic Review, 78(5):936-953, 1988.

[6] J. Yurkiewicz. Software survey: Forecasting 2000. OR/MS Today, 27(1), 2000. 